\title{
Analysis of the autoimmune response against BP180 in patients with Alzheimer's disease
}

\author{
Ya-Nan Wang ${ }^{1}$, Christoph M. Hammers ${ }^{2}$, Xuming Mao ${ }^{3}$, Hong-Zhong Jin ${ }^{1}, \mathrm{Jing}_{\mathrm{Yuan}}{ }^{4}, \mathrm{Li} \mathrm{Li}^{1} \wedge$ \\ ${ }^{1}$ Department of Dermatology, Peking Union Medical College Hospital, Chinese Academy of Medical Sciences \& Peking Union Medical College, \\ Beijing, China; ${ }^{2}$ Department of Dermatology, University of Luebeck, Luebeck, Germany; ${ }^{3}$ Department of Dermatology, University of Pennsylvania, \\ Philadelphia, PA, USA; ${ }^{4}$ Department of Neurology, Peking Union Medical College Hospital, Chinese Academy of Medical Sciences \& Peking Union \\ Medical College, Beijing, China \\ Contributions: (I) Conception and design: YN Wang, L Li; (II) Administrative support: HZ Jin, J Yuan, L Li; (III) Provision of study materials or \\ patients: J Yuan, L Li; (IV) Collection and assembly of data: YN Wang, J Yuan; (V) Data analysis and interpretation: YN Wang, CM Hammers, X \\ Mao; (VI) Manuscript writing: All authors; (VII) Final approval of manuscript: All authors. \\ Correspondence to: Dr. Li Li; Dr. Jing Yuan. No. 1 Shuai Fu Yuan Street, Beijing, China. Email: lilipumch2007@sina.com; yuanjing@pumch.cn.
}

Background: Current epidemiological studies suggest a significant correlation between Alzheimer's disease (AD) and bullous pemphigoid (BP). However, the autoimmune response against BP180 in patients with AD has not been fully understood. investigated.

Methods: We randomly enrolled 48 patients with $\mathrm{AD}$ and 50 sex- and age-matched healthy controls. We detected the presence/absence and the level of anti-BP180/BP230 immunoglobulin G (IgG) autoantibodies in the patients' serum to determine whether said antibodies possess reactivity against the BP180 protein in the human brain and skin.

Results: The enzyme-linked immunosorbent assay (ELISA) results revealed that the positive rate of antiBP180 autoantibodies in patients with AD (23/48, 47.9\%) was significantly higher than that in controls (4/50, 8.0\%; $\mathrm{P}<0.0001)$. These ELISA-positive patients were further examined through immunoblotting. Sera from nine patients with $\mathrm{AD}(9 / 23,39.1 \%)$ and one control $(1 / 4,25.0 \%)$ reacted with human cutaneous recombinant full-length BP180 and BP180-noncollagenous 16A (NC16A). Sera from 11 (11/23, 47.8\%) patients with $\mathrm{AD}$ reacted with a $180-\mathrm{kDa}$ protein from the human brain extract, but none of the controls' sera recognized the corresponding protein band. The majority of the patients in the anti-BP180-positive AD group were men $(14 / 23,60.9 \%)$ who were older (74.0 years) compared with those in the control group $(6 / 25$, $24.0 \% ; \mathrm{P}<0.05)(72.2$ years; $\mathrm{P}<0.01)$.

Conclusions: Anti-BP180 autoantibodies are present in AD and recognize human recombinant full-length BP180 and a 180-kDa protein from the human brain extract, suggesting that BP180 is a shared autoantigen in $\mathrm{AD}$ and $\mathrm{BP}$ and may help clarify the mechanism to explain why a high risk of $\mathrm{BP}$ exists in AD. Elderly male patients with $\mathrm{AD}$ are significantly more likely to develop BP180 serum autoreactivity compared with other patients with $\mathrm{AD}$.

Keywords: Anti-BP180 autoantibodies; BP180; BP180-NC16A; Alzheimer's disease (AD); bullous pemphigoid (BP)

Submitted Jul 21, 2020. Accepted for publication Oct 30, 2020.

doi: $10.21037 /$ atm-20-5343

View this article at: http://dx.doi.org/10.21037/atm-20-5343

^ ORCID: Ya-Nan Wang, 0000-0002-6434-7965; Li Li, 0000-0002-8280-7291. 


\section{Introduction}

Bullous pemphigoid (BP) is the most common autoimmune bullous disorder and mainly affects elderly people (1). Compared with people aged under 60 years, those aged over 90 years have a 300-fold increased risk of BP (2). $\mathrm{BP}$ is characterized by autoantibodies against two hemidesmosome proteins [collagen XVII (BP180 or BP antigen 2) and dystonin-e (BP230 or BP antigen 1)] of the skin and mucous membranes $(3,4)$. Notably, BP180 is expressed not only in basal keratinocytes but also in the nucleus basalis and hippocampus of the brain, which is exactly where Alzheimer's disease (AD)-related lesions were observed (5). Alternative splicing and variation in the translational start site of the BP180 gene result in the expression of skin- or neuron-specific isoforms $(5,6)$. In $\mathrm{BP}$, autoantibodies against BP180 are pathogenic and associated with disease activity, especially at diagnosis and outbreak $(4,7)$. Autoantibodies that bind to extracellular noncollagenous 16A (NC16A) account for the majority and play a key role in pathogenesis $(7,8)$. However, most patients also have immunoglobulin G ( $\operatorname{IgG}$ ) antibodies against epitopes outside the NC16A domain (9). Autoantibodies against BP230 can also be detected in the majority of patients with $\mathrm{BP}$, although their pathogenic role remains controversial (10).

Several epidemiological studies have suggested that $\mathrm{AD}$ is often comorbid with $\mathrm{BP}$ (11-13). AD is a neurodegenerative disorder of uncertain cause and pathogenesis that primarily affects older adults; furthermore, it is the most common cause of dementia (14). The hallmark neuropathologic changes of $\mathrm{AD}$ are extracellular amyloid $\beta$ deposition and phosphorylated Tau protein, pathologically marked by diffuse and neuritic plaques and neurofibrillary tangles (15). Patients aged older than 80 years with a neurological disease (ND) were 10 times more likely to have BP compared with those without, and the most frequently associated conditions were dementia and cerebral stroke (16). Yu Phuan et al. (17) found that $73.8 \%$ (76/103) of patients with BP had a history of at least one pre-existing ND, among which only dementia was statistically significant $(\mathrm{P}<0.05)$. In a systematic review, patients with BP had an increased risk of dementia [odds ratio (OR): 5.48 (95\% CI: 3.26-9.23); $\mathrm{P}<0.001]$ and ND preceded $\mathrm{BP}$ in the majority of cases with a mean time interval of 6.7 years (18). According to our study and others, the development of BP autoantibodies against both the skin and neuronal forms of antigens in BP with ND might be related to cutaneous- and neuronalsubtype BP antigens that have different locations, epitopes, and natural conformations (19-22). In this study, we randomly enrolled 48 patients with $\mathrm{AD}$ and 50 sex- and agematched healthy controls to detect the level of anti-BP180/ BP230 IgG autoantibodies in sera. We sought to determine whether these antibodies have reactivity against the BP180 protein of the human brain and skin, which may help reveal the mechanism of high $\mathrm{BP}$ incidence in $\mathrm{AD}$.

We present the following article in accordance with the MDAR reporting checklist (available at http://dx.doi. org/10.21037/atm-20-5343).

\section{Methods}

\section{Patient samples}

Forty-eight patients with AD were recruited from the Neurology Clinics of Peking Union Medical College Hospital. AD was diagnosed based on medical history, clinical symptoms, physical examination (including nervous system), neuropsychological assessment, laboratory examination, and imaging examination in the Department of Neurology (Table 1). Age- and sex-matched healthy controls were recruited from patients undergoing benign neoplasm resection in the Department of General Surgery (Table 1). Patients with NDs (stroke, Parkinson's disease, other subtypes of dementia, multiple sclerosis) and skin diseases (only bullous disease, dermatitis, eczema, and other autoimmune diseases) were excluded. The normal human brain tissue was obtained from donors undergoing temporal lobe resection for medication-resistant epilepsy treatment (normal brain tissues adjacent to the focuses, as confirmed by a neuropathologist).

The study was conducted in accordance with the Declaration of Helsinki (as revised in 2013). The study was reviewed and approved by IRB members of Peking Union Medical College Hospital, CAMS \& PUMCA (approval number S-300). All patients enrolled completed the informed consent form.

\section{Enzyme-linked immunosorbent assay (ELISA)}

The anti-BP180/BP230 IgG autoantibodies in serum samples were determined using a human BP180 (NC16A domains) IgG quantification kit (MEASACUP BP180 TEST, MBL, Nagoya, Japan) and human BP230 (N-and C-terminal domains) IgG quantification kit (BP230 ELISA Kit, MBL) according to the manufacturer's instructions (based on a cutoff value of $>9 \mathrm{U} / \mathrm{mL}$ ). Briefly, the standard 
Table 1 Characteristics and immunological results of patients with AD and controls

\begin{tabular}{|c|c|c|c|}
\hline Variable & $A D$ & Control & $P$ value \\
\hline $\operatorname{Sex}(M / F)$ & $20 / 28$ & $21 / 29$ & NS \\
\hline $\mathrm{Age}^{\dagger}$ & $72.5 \pm 6.6$ & $72.8 \pm 7.2$ & NS \\
\hline BP180 ELISA, +/- & $23 / 25$ & $4 / 46$ & $<0.0001$ \\
\hline Values of BP180 autoantibody-positive patients $(\mathrm{U} / \mathrm{mL})^{\dagger, \neq}$ & $22.85 \pm 20.94$ & $12.94 \pm 3.04$ & $<0.05$ \\
\hline Human cutaneous BP180, +/- & $9 / 14$ & $1 / 3$ & NS \\
\hline Human cutaneous NC16A, +/- & $9 / 14$ & $1 / 3$ & NS \\
\hline Human brain extract (180 kD), +/- & $11 / 12$ & $0 / 4$ & NS \\
\hline Values of BP230 autoantibody-positive patients $(\mathrm{U} / \mathrm{mL})^{\dagger, \neq}$ & $21.26 \pm 16.90$ & $13.03 \pm 2.78$ & $<0.05$ \\
\hline Human cutaneous extract (230 kD), +/- & $0 / 16$ & $0 / 11$ & NS \\
\hline Human brain extract (230 kD), +/- & $5 / 11$ & $0 / 11$ & NS \\
\hline IIF (salt split), +/- & $0 / 31$ & $0 / 14$ & NS \\
\hline
\end{tabular}

${ }^{\dagger}$, data given as mean \pm standard deviation; ${ }^{\ddagger}$, based on a cut-off value $>9 \mathrm{U} / \mathrm{mL}$. AD, Alzheimer's disease; ELISA, enzyme-linked immunosorbent assay; IIF, indirect immunofluorescence; NS, not significant.

serum and patient serum were added to a microporous strip embedded with BP180/BP230 antigens. Human IgG antibodies labelled with horseradish peroxide (HRP) and peroxidase substrate were added successively. Subsequently, acid solution was added to terminate the enzyme reaction and stabilize the color reaction. Finally, the absorbance was determined using a spectrophotometer (Genova Nano, Jenway, Stone, UK).

\section{Western blotting}

The recombination of human cutaneous full-length BP180 and BP180-NC16A and western blotting were performed as previously described (21). Briefly, primers were designed according to the full length BP180/NC16A cDNA sequence to amplify the target gene fragments by PCR and sequence for FLAG tag was incorporated into the cDNA. Then the human full length BP180/NC16A gene fragments were subcloned into the pcDNA3.1 mammalian expression vector. HEK 293 cells were transiently transfected with the plasmids and lipofectamine (Life Technologies, Carlsbad, CA, USA) as per the manufacturer's instructions, followed by lysis of the cells in a lysis buffer [50 $\mathrm{mM}$ Tris
(PH8.0), $300 \mathrm{mM} \mathrm{NaCl}, 1 \%$ Triton X-100, $1 \mathrm{mM}$ DTT, $5 \%$ glycerol]. The proteins in lysates were purified using FLAG peptide affinity chromatography and peptide elution. Expression of the target proteins were confirmed by western-blot (anti-FLAG tag antibody) and quantified by the protein quantification assay kit. Human brain samples $(5 \mathrm{~mm})$ were lysed in RIPA buffer with a protease inhibitor (Beyotime, Shanghai, China) and quantified using a BCA assay kit (Beyotime). Proteins (10 $\mu \mathrm{g}$ per lane) were separated with $8 \%$ SDS-PAGE and transferred onto a polyvinylidene fluoride membrane (Millipore, Billerica, MA, USA). Then, the membrane was incubated with human serum samples $(0.5 \mu \mathrm{g} / \mu \mathrm{L})$ and anti-human $\lg \mathrm{G}$ HRP (1:2,500) (Abcam, Cambridge, UK). Finally, bands were visualized using a detection solution (Merck KGaA, Darmstadt, Germany) and exposed using an image analysis system (Tanon, Shanghai, China). Anti-human BP180/ BP230 antibodies (Abcam) were used as the positive control. $5.0 \%$ defatted milk powder was used as the negative control.

\section{Salt-split skin indirect immunofluorescence (SSS-IIF)}

SSS-IIF was performed as previously described (21). In 
brief, $5-\mu \mathrm{m}$ frozen nonfixed sections of the human skin (treated with $1 \mathrm{M} \mathrm{NaCl})$ were blocked $(1 \%$ bovine serum albumin in phosphate-buffered saline) and incubated with patients' serum at 1:4-1:320 dilution with rabbit anti-human IgG-FITC (Abcam), followed by routine indirect immunofluorescence microscopy. Clear linear immunostaining of the epidermal side of the basement membrane zone (BMZ) was considered positive, whereas no fluorescence was considered negative.

\section{Statistical analysis}

All the experiments were replicated at least three times. Original data were analyzed using SPSS 22.0 (IBM SPSS, Leawood, KS, USA) and are represented as the mean \pm SD. Different statistical methods were used according to the type of data. Student's $t$-tests (bilateral) were used to analyze the normally distributed continuous variables, such as age and BP180/BP230 antibody titer. The chi-squared test was used to analyze qualitative variables, such as BP180 antibody (positive/negative) and sex (male/female). $\mathrm{P}<0.05$ was considered to represent statistical significance. GraphPad Prism 8.0 (GraphPad Prism, San Diego, CA, USA) was used to compile figures.

\section{Results}

We randomly enrolled 48 patients with $\mathrm{AD}$ and 50 sex- and age-matched healthy controls to examine the level of antiBP180/BP230 IgG autoantibodies in sera. Using ELISA, we found that $47.9 \%(23 / 48)$ of patients in the $\mathrm{AD}$ group and only $8.0 \%(4 / 50, \mathrm{P}<0.0001)$ of the controls had anti-BP 180 autoantibodies (Table 1). Anti-BP180 autoantibody titers in the $\mathrm{AD}$ group were higher $(13.08 \pm 17.23 \mathrm{U} / \mathrm{mL})$ than those in the control group $(5.78 \pm 4.24 \mathrm{U} / \mathrm{mL} ; \mathrm{P}<0.01)$ (Table 1). Moreover, the titers of BP180 autoantibody-positive patients with $\mathrm{AD}(22.85 \pm 20.94 \mathrm{U} / \mathrm{mL})$ were significantly higher than those of controls $(12.94 \pm 3.04 \mathrm{U} / \mathrm{mL} ; \mathrm{P}<0.05$; Table 1). In addition, the positive rate of anti-BP230 autoantibodies in the $\mathrm{AD}$ group $(16 / 48,33.3 \%)$ was higher than that in the control group $(11 / 50,22.0 \% ; \mathrm{P}=0.26)$ according to the ELISA (Table 1). Anti-BP230 autoantibody titers in the $\mathrm{AD}$ group $(9.60 \pm 13.00 \mathrm{U} / \mathrm{mL})$ were also higher than those in the control group $(5.96 \pm 4.37 \mathrm{U} / \mathrm{mL} ; \mathrm{P}=0.06)$. However, differences in anti-BP230 antibodies between the patient and control groups were statistically nonsignificant (Table 1).

Next, to determine whether these anti-BP180 IgG positive antibodies (23 AD samples and four healthy controls) had reactivity against the recombinant full-length BP180/NC16A and the BP180 protein in the human brain, we performed Western blotting against the human brain extract and human recombinant full-length BP180 and BP180-NC16A. We used the normal brain tissue from patients with epilepsy because temporal lobe resection where hippocampus located in is a common appropriate treatment for patients with difficult-to-treat focal epilepsy. The results indicated that sera from nine of the 23 patients with $\mathrm{AD}(9 / 23,39.1 \%)$ and one healthy control $(1 / 4,25.0 \%)$ appeared to recognize human cutaneous recombinant fulllength BP180 and BP180-NC16A (Figure 1A,B, Table 1). Notably, sera from $11(11 / 23,47.8 \%)$ patients with $A D$ exhibited reactivity against a $180-\mathrm{kDa}$ protein of the human brain extract, but none of the controls' sera recognized the corresponding protein band (Figure 1C, Table 1). Sera from five patients with $\mathrm{AD}$ reacted with both a $180-\mathrm{kDa}$ band of the human brain extract and human recombinant fulllength BP180 and BP180-NC16A.

Anti-BP180/BP230-positive sera were examined through SSS-IIF, and no epidermal side fluorescence signals were observed in the BMZ (Table 1). Furthermore, no BP-like skin lesions were noted in the $\mathrm{AD}$ or control group during the 3-year follow-up, indicating that the appearance of serum BP180 autoantibodies is not associated with the clinical onset of BP during short-term follow-up.

Subsequently, we compared the characteristics of antiBP180-positive and -negative patients with $\mathrm{AD}$. The results revealed that compared with the anti-BP180-negative $\mathrm{AD}$ group, the positive group had more male (14/23 vs. 6/25, $60.9 \%$ vs. $24.0 \% ; \mathrm{P}<0.05)$ and older patients $(74.0$ vs. 72.2 years; $\mathrm{P}<0.01)$, suggesting that elderly male patients with $\mathrm{AD}$ may be more likely to exhibit an autoimmune antiBP180 serum response.

\section{Discussion}

BP180 is expressed not only in basal keratinocytes but also in the nucleus basalis and hippocampus of the brain (6). Coincidentally, the nucleus basalis of Meynert and the hippocampal region, which exhibit strong BP180 expression, are well-recognized predilection areas for AD-related lesions (5). Specifically, BP180 is located in lipofuscin granules, which are also associated with $\mathrm{AD}$ and ageing processes $(23,24)$. Our data demonstrated that anti-BP180 autoantibodies are more frequent in AD. In a study by Kokkonen (25), 18.0\% (20/115) of patients 


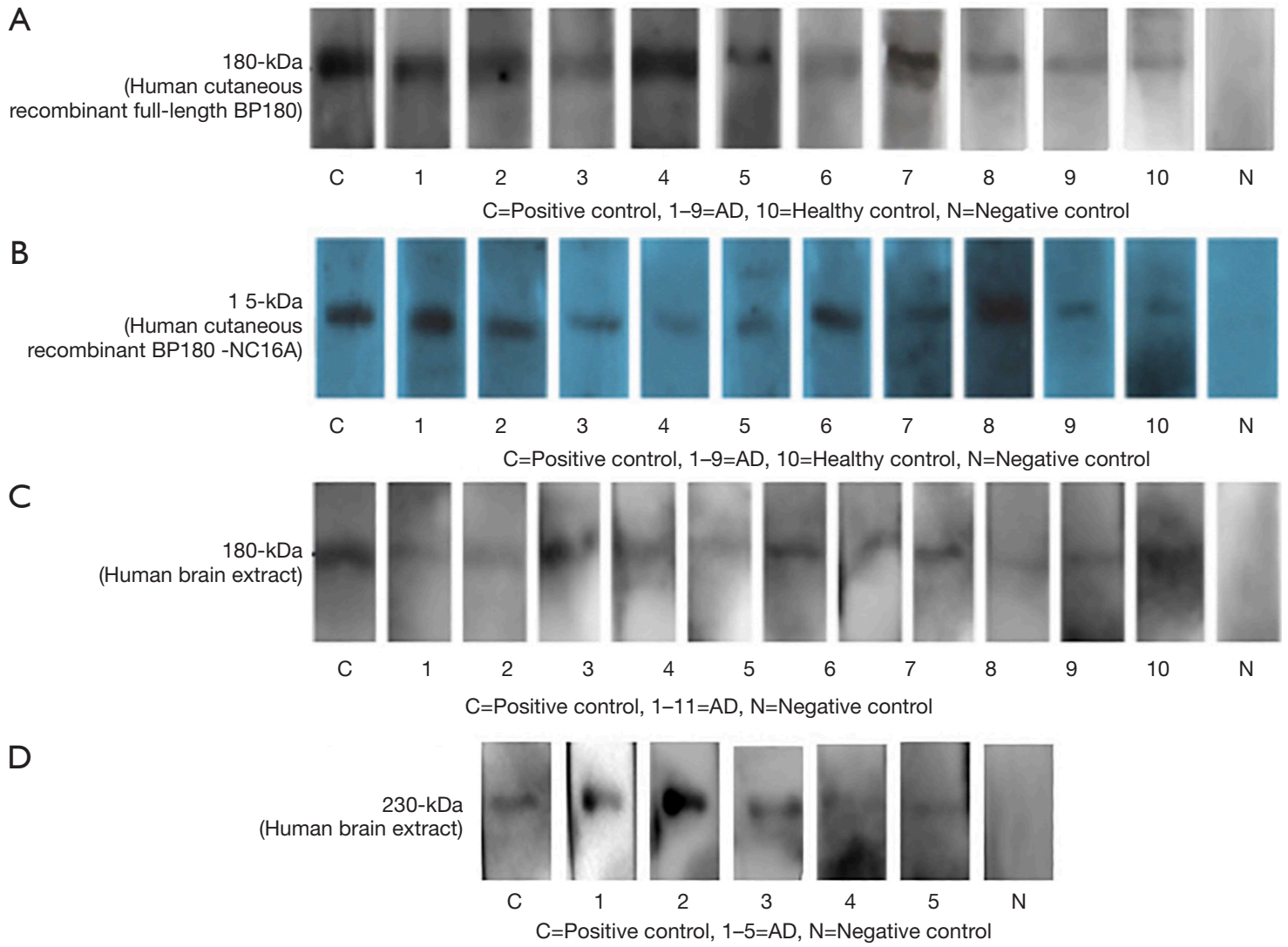

Figure 1 Detection of anti-BP180 antibodies in AD or control sera by immunoblotting. (A,B) 9 out of 23 AD samples and one healthy control sample reacted with human recombinant full-length BP180 and BP180-NC16A. (C) 11 out of 23 AD samples recognized a 180-kDa protein from human brain extract. (D) 5 out of $16 \mathrm{AD}$ samples recognized a $230-\mathrm{kDa}$ protein from human brain extract. C, positive control antibodies (anti-BP180/230 antibodies and sera from a patient with BP); N, negative controls (5.0\% defatted milk powder); AD, Alzheimer's disease.

with $\mathrm{AD}$ were positive for $\mathrm{BP} 180$, which was close to our current result $(9 / 48,18.8 \%)$. Furthermore, we found that $18.8 \%(9 / 48)$ of AD samples reacted with BP180-NC16A, the major epitope of dermal BP180, which revealed that these autoantibodies found in patients with $\mathrm{AD}$ could also react with BP180-NC16A similar to in patients with BP. Moreover, different ELISA results have been reported in several studies. For example, Foureur et al. (26) tested BP180 antibodies in 138 patients aged over 69 years with no signs of BP (69 patients with dementia and 60 nondementia controls) and found BP180 autoantibodies in 7\% (9/138) of dementia patients, which was obviously lower than the proportion in our study. In Messingham's study (27), only one of $26(3.8 \%)$ patients with different types of dementia had detectable autoantibodies against NC16A. The differing results of these studies may be explained by methodological differences, different inclusion criteria, different sample sizes, or different average ages (25).

In the present study, we observed that the serum samples of anti-BP180 antibody-positive patients with $\mathrm{AD}$ recognized a $180-\mathrm{kDa}$ protein of the human brain extract as well as human cutaneous BP180 and BP180-NC16A, which increases the likelihood that BP180 indeed serves as a common autoantigen in $\mathrm{AD}$ and BP. Low titers of anti-BP180 autoantibodies may be biomarkers for BP at a later stage. In a previous study, we found that the $180-\mathrm{kDa}$ proteins of mouse and human brains can be recognized by the serum samples of patients with BP + ND $(19,20)$. Therefore, the current study adds data to the hypothesis that $\mathrm{BP}$ antibodies in patients with $\mathrm{AD}$ could cross-react with antigens in both the skin and brain. In our studies, BP180 immunoblotting revealed a single band of $180 \mathrm{kDa}$ in the normal human brain extract, which was the same with the molecular weights of keratinocytes. By contrast, 
Seppänen (6) found that BP180 immunoblotting revealed a single band of $150 \mathrm{kDa}$ in the post-mortem human brain extract, which may be because of the partial degradation of the protein during post-mortem delay (6). Besides, sera from five patients with $\mathrm{AD}$ reacted with a $230-\mathrm{kDa}$ band of the human brain extract (Figure 1D). Yang et al. (28) found the weight of BPAG1n (neuro isoform of BP230) was $280-\mathrm{kDa}$, and a $\sim 250-\mathrm{kDa}$ unknown band was also detected. Moreover, approx. $10 \%$ molecular weight bias is usually difficult to avoid in immunoblotting. Therefore, the 230$\mathrm{kDa}$ protein present in the brain extract in our study may be identical with the $\sim 250-\mathrm{kDa}$ band in the study of Yang et al. (28) or the degraded BPAG1n in brain. The positive rate of anti-BP180 antibody of ELISA was higher than that of immunoblotting in our study (Table 1). A metaanalysis described ELISA as a quantitative test with high sensitivity and specificity (87\% and $98-100 \%$, respectively) for diagnosis of BP (29), while immunoblotting was a rather semi-quantitative test, potentially explaining ELISA positive patients with negative immunoblotting results.

None of the individuals exhibited IgG reactivity against the BMZ by SSS-IIF in our study. Consistently, no patients with $\mathrm{AD}$ or controls exhibited BP-like skin lesions during the 3-year follow-up, which is consistent with the findings of studies conducted by Kokkonen (25) and Messingham (27) in $\mathrm{AD}$ and Parkinson's disease. All these negative results from our study and above published researches suggest that autoantibodies found in patients with $\mathrm{AD}$ may be from the central nervous system and not identical to those in BP patients. This likely has several causes, the first of which is distinct epitopes. The autoantibodies in $\mathrm{AD}$ tend to recognize BP180 in its native conformation rather than its denatured conformation (22). Second, the titers of antibodies may be too low to develop BP skin lesions. In $\mathrm{BP}$, autoantibody titers are markers for disease activity and fluctuate with disease severity (9). Third, some patients might be misdiagnosed because of the early-stage, atypical, nonbullous variants of $\mathrm{BP}$, such as excoriated, eczematous, or urticarial lesions $(30,31)$. Fourth, according to a systematic review of epidemiological studies, ND preceded $\mathrm{BP}$ in the majority of cases with a mean time interval of 6.7 years (18). We speculated that the sample size may be not large enough, and the follow-up period may be not long enough to develop BP-like skin lesions. In the future, a larger sample size and longer follow-up should help further elucidate the relationship between BP autoantibody development and BP-like skin lesions.

Based on these observations, we speculated that damage or alterations in the human central nervous system during the course of $\mathrm{AD}$ may expose neuronal isoform of BP180 in brain, thereby triggering an immune reaction that, along with immunological cross-reactions, determines future cutaneous damage $(19,20)$. On the one hand, the activation of neuroinflammation in $\mathrm{AD}$ has been confirmed by many studies, which is both a cause and a consequence of $\mathrm{AD}(32,33)$. Chronic neuroinflammation in $\mathrm{AD}$ or ageing individuals activates the peripheral immune system to eliminate $A \beta$ (34). In addition, a weakened blood-brain barrier permeability in $\mathrm{AD}$ supports the adaptive immune response $(32,35)$. On the other hand, it was reported that the positive rate of anti-brain antibodies in $\mathrm{AD}$ was significantly higher than that of normal people (36). Animal models showed that vaccination with $A \beta 1-42$ produces a Th2 response that encourages $B$ cells to produce anti-A $\beta$ antibodies (35). As previously described, BP180 expression and $\mathrm{A} \beta$ deposition could occur in the same regions of the brain. All these findings prompted us to speculate that the neuronal isoform of BP antigen act as a shared autoantigen in both $\mathrm{AD}$ and $\mathrm{BP}$. It is possible that alterations in the central nervous system in the course of $\mathrm{AD}$ could expose the neuronal isoform of $\mathrm{BP}$ antigen, triggering an immune reaction that, along with the immunological cross-reactions, results in the development of $\mathrm{BP}(19,20)$. However, this supposition requires further experiments for verification.

In summary, anti-BP180 autoantibodies exist in patients with $\mathrm{AD}$ and recognize a $180-\mathrm{kDa}$ protein of human brain extract as well as human cutaneous BP180 and BP180NC16A. These observations raise the possibility that BP 180 acts as a shared autoantigen in both $\mathrm{AD}$ and BP. In addition, our study suggests that old age and male sex may be risk factors for developing BP antibodies in AD. Future studies using longer-term clinical follow-ups as well as animal or cell models may help to further clarify why BP is significantly associated with NDs.

\section{Acknowledgments}

We would like to thank the Prof. Nan Yang, Department of Pharmacology, Institute of Basic Medical Sciences, Chinese Academy of Medical Sciences, School of Basic Medicine, Peking Union Medical College, for her outstanding assistance.

Funding: this work was supported by the National Key Research and Development Program of China (No. 2016YFC0901500), National Natural Science Foundation of China (81972945), Milstein Medical Asian American 
Partnership foundation (2017, Dermatology) and the Education Reform Projects of Peking Union Medical College (No. 2016zlgc0106).

\section{Footnote}

Reporting Checklist: The authors have completed the MDAR reporting checklist. Available at http://dx.doi.org/10.21037/ atm-20-5343

Data Sharing Statement: Available at http://dx.doi. org/10.21037/atm-20-5343

Conflicts of Interest: All authors have completed the ICMJE uniform disclosure form (available at http://dx.doi. org/10.21037/atm-20-5343). The authors have no conflicts of interest to declare.

Ethics Statement: The authors are accountable for all aspects of the work in ensuring that questions related to the accuracy or integrity of any part of the work are appropriately investigated and resolved. The study was conducted in accordance with the Declaration of Helsinki (as revised in 2013). The study was reviewed and approved by IRB members of Peking Union Medical College Hospital, CAMS \& PUMCA (approval number S-300). All patients enrolled completed the informed consent form.

Open Access Statement: This is an Open Access article distributed in accordance with the Creative Commons Attribution-NonCommercial-NoDerivs 4.0 International License (CC BY-NC-ND 4.0), which permits the noncommercial replication and distribution of the article with the strict proviso that no changes or edits are made and the original work is properly cited (including links to both the formal publication through the relevant DOI and the license). See: https://creativecommons.org/licenses/by-nc-nd/4.0/.

\section{References}

1. Egami S, Yamagami J, Amagai M. Autoimmune bullous skin diseases, pemphigus and pemphigoid. J Allergy Clin Immunol 2020;145:1031-47.

2. Jung M, Kippes W, Messer G, et al. Increased risk of bullous pemphigoid in male and very old patients: A population-based study on incidence. J Am Acad Dermatol 1999;41:266-8.

3. Kridin K, Ludwig RJ. The Growing Incidence of Bullous
Pemphigoid: Overview and Potential Explanations. Front Med (Lausanne) 2018;5:220.

4. Hammers CM, Stanley JR. Mechanisms of Disease: Pemphigus and Bullous Pemphigoid. Annu Rev Pathol 2016;11:175-97.

5. Seppänen A. Collagen XVII: a shared antigen in neurodermatological interactions? Clin Dev Immunol 2013;2013:240570.

6. Seppänen A, Suuronen T, Hofmann SC, et al. Distribution of collagen XVII in the human brain. Brain Res 2007;1158:50-6.

7. Liu Y, Li L, Xia Y. BP180 Is Critical in the Autoimmunity of Bullous Pemphigoid. Front Immunol 2017;8:1752.

8. Di Lernia V, Casanova DM, Goldust $M$, et al. Pemphigus Vulgaris and Bullous Pemphigoid: Update on Diagnosis and Treatment. Dermatol Pract Concept 2020;10:e2020050.

9. Bağc1 IS, Horváth ON, Ruzicka T, et al. Bullous pemphigoid. Autoimmun Rev 2017;16:445-55.

10. Kunzli K, Favre B, Chofflon M, et al. One gene but different proteins and diseases: the complexity of dystonin and bullous pemphigoid antigen 1. Exp Dermatol 2016;25:10-6.

11. Khosravani S, Handjani F, Alimohammadi R, et al. Frequency of Neurological Disorders in Bullous Pemphigoid Patients: A Cross-Sectional Study. Int Sch Res Notices 2017;2017:6053267.

12. Pietkiewicz P, Gornowicz-Porowska J, BowszycDmochowska M, et al. Bullous pemphigoid and neurodegenerative diseases: a study in a setting of a Central European university dermatology department. Aging Clin Exp Res 2016;28:659-63.

13. Lai YC, Yew YW, Lambert WC. Bullous pemphigoid and its association with neurological diseases: a systematic review and meta-analysis. J Eur Acad Dermatol Venereol 2016;30:2007-15.

14. Ballard C, Gauthier S, Corbett A, et al. Alzheimer's disease. Lancet 2011;377:1019-31.

15. Graham WV, Bonito-Oliva A, Sakmar TP. Update on Alzheimer's Disease Therapy and Prevention Strategies. Annu Rev Med 2017;68:413-30.

16. Jedlickova H, Hlubinka M, Pavlik T, et al. Bullous pemphigoid and internal diseases - A case-control study. Eur J Dermatol 2010;20:96-101.

17. Yu Phuan CZ, Yew YW, Tey HL. Bullous pemphigoid and antecedent neurological diseases: An association with dementia. Indian J Dermatol Venereol Leprol 2017;83:457-61. 
18. Milani-Nejad N, Zhang M, Kaffenberger J. The association between bullous pemphigoid and neurological disorders: a systematic review. Eur J Dermatol 2017;27:472-81.

19. Li L, Chen J, Wang B, et al. Sera from patients with bullous pemphigoid (BP) associated with neurological diseases recognized $\mathrm{BP}$ antigen 1 in the skin and brain. $\mathrm{Br}$ J Dermatol 2009;160:1343-5.

20. Chen J, Li L, Chen J, et al. Sera of elderly bullous pemphigoid patients with associated neurological diseases recognize bullous pemphigoid antigens in the human brain. Gerontology 2011;57:211-6.

21. Wang Y, Mao X, Wang D, et al. Anti-BP180 Autoantibodies Are Present in Stroke and Recognize Human Cutaneous BP180 and BP180-NC16A. Front Immunol 2019;10:236.

22. Tuusa J, Lindgren O, Tertsunen HM, et al. BP180 Autoantibodies Target Different Epitopes in Multiple Sclerosis or Alzheimer's Disease than in Bullous Pemphigoid. J Invest Dermatol 2019;139:293-9.

23. Seppanen A, Miettinen R, Alafuzoff I. Neuronal collagen $\mathrm{XVII}$ is localized to lipofuscin granules. Neuroreport 2010;21:1090-4.

24. Keller JN. Age-related neuropathology, cognitive decline, and Alzheimer's disease. Ageing Res Rev 2006;5:1-13.

25. Kokkonen N, Herukka SK, Huilaja L, et al. Increased Levels of the Bullous Pemphigoid BP180 Autoantibody Are Associated with More Severe Dementia in Alzheimer's Disease. J Invest Dermatol 2017;137:71-6.

26. Foureur N, Mignot S, Senet P, et al. Correlation between the presence of type-2 anti-pemphigoid antibodies and dementia in elderly subjects with no clinical signs of pemphigoid. Ann Dermatol Venereol 2006;133:439-43.

27. Messingham KA, Aust S, Helfenberger J, et al.

Cite this article as: Wang YN, Hammers CM, Mao X, Jin HZ, Yuan J, Li L. Analysis of the autoimmune response against BP180 in patients with Alzheimer's disease. Ann Transl Med 2021;9(2):107. doi: 10.21037/atm-20-5343
Autoantibodies to Collagen XVII Are Present in Parkinson's Disease and Localize to Tyrosine-Hydroxylase Positive Neurons. J Invest Dermatol 2016;136:721-3.

28. Yang Y, Dowling J, Yu QC, et al. An essential cytoskeletal linker protein connecting actin microfilaments to intermediate filaments. Cell 1996;86:655-65.

29. Muglia C, Bronsnick T, Kirkorian AY, et al. Questioning the specificity and sensitivity of ELISA for bullous pemphigoid diagnosis. Cutis 2017;99:E27-e30.

30. Bernard P, Antonicelli F. Bullous Pemphigoid: A Review of its Diagnosis, Associations and Treatment. Am J Clin Dermatol 2017;18:513-28.

31. Chou PS, Chou TC, Chang CH, et al. Chronic eczematous dermatitis in patients with neurodegenerative diseases may be an early marker of bullous pemphigoid. Med Hypotheses 2017;103:86-9.

32. Zhang ZG, Li Y, Ng CT, et al. Inflammation in Alzheimer's Disease and Molecular Genetics: Recent Update. Arch Immunol Ther Exp (Warsz) 2015;63:333-44.

33. Pimplikar SW. Neuroinflammation in Alzheimer's disease: from pathogenesis to a therapeutic target. J Clin Immunol 2014;34 Suppl 1:S64-9.

34. Wieland CN, Comfere NI, Gibson LE, et al. Antibullous pemphigoid 180 and 230 antibodies in a sample of unaffected subjects. Arch Dermatol 2010;146:21-5.

35. Butchart J, Holmes C. Systemic and central immunity in Alzheimer's disease: therapeutic implications. CNS Neurosci Ther 2012;18:64-76.

36. Rotman M, Welling MM, Bunschoten A, et al. Enhanced glutathione PEGylated liposomal brain delivery of an anti-amyloid single domain antibody fragment in a mouse model for Alzheimer's disease. J Control Release 2015;203:40-50. 